\title{
On forbidden poset problems in the linear lattice
}

\author{
Jimeng Xiao* \\ Department of Applied Mathematics \\ Northwestern Polytechnical University \\ Xi'an-Budapest Joint Research Center for Combinatorics \\ Xi'an, P.R. China
}

Alfréd Rényi Institute of Mathematics, Hungarian Academy of Sciences

Budapest, Hungary

xiaojimeng@mail.nwpu.edu.cn

Casey Tompkins ${ }^{\dagger}$

Department of Mathematics

Karlsruhe Institute of Technology

Karlsruhe, Germany

ctompkins496@gmail.com

Submitted: Jun 30, 2019; Accepted: Nov 8, 2019; Published: Jan 24, 2020

(C) The authors. Released under the CC BY-ND license (International 4.0).

\begin{abstract}
In this note, we determine the maximum size of a $\left\{\mathrm{V}_{k}, \Lambda_{l}\right\}$-free family in the lattice of vector subspaces of a finite vector space both in the non-induced case as well as the induced case, for a large range of parameters $k$ and $l$. These results generalize earlier work by Shahriari and Yu. We also prove a general LYM-type lemma for the linear lattice which resolves a conjecture of Shahriari and Yu.
\end{abstract}

Mathematics Subject Classifications: 05D05

\section{Introduction}

Given partially ordered sets (posets) $P$ and $Q$, we say that $P$ is a subposet of $Q$ if there exists an injection $\phi: P \rightarrow Q$ such that $x \leqslant_{P} y$ implies $\phi(x) \leqslant_{Q} \phi(y)$. If we also have that $\phi(x) \leqslant_{Q} \phi(y)$ implies $x \leqslant_{P} y$, then we say $P$ is an induced subposet of $Q$. Viewing collections of sets as posets under the inclusion relation, we have the following extremal

*Supported by the National Research, Development and Innovation Office NKFIH, grant K116769, by CSC (No. 201706290171) and NSFC (No. 11671320).

${ }^{\dagger}$ Supported by IBS-R029-C1. 
functions, first introduced by Katona and Tarján [10]. For any collection of finite posets $\mathcal{P}$, let $\mathrm{La}(n, \mathcal{P})$ be the maximum size of a family of subsets of $\{1,2, \ldots, n\}$ which does not contain any $P \in \mathcal{P}$ as a subposet, and let $\operatorname{La}^{*}(n, \mathcal{P})$ be the maximum size of a family of subsets of $\{1,2, \ldots, n\}$ which does not contain any $P \in \mathcal{P}$ as an induced subposet. In the case $\mathcal{P}=\{P\}$ for some poset $P$, we instead write simply $\operatorname{La}(n, P)$ and $\operatorname{La}^{*}(n, P)$. We denote the sum of the $k$ largest binomial coefficients of the form $\left(\begin{array}{l}n \\ i\end{array}\right)$ by $\Sigma(n, k)$.

Let $V$ be an $n$-dimensional vector space over a finite field $\mathbb{F}_{q}$, where $q$ is a prime power. The linear lattice of dimension $n$ is the poset of subspaces of $V$ under the inclusion relation. We denote by $\left[\begin{array}{l}V \\ k\end{array}\right]_{q}$ the set of all $k$-dimensional subspaces of $V$ (this set is often referred to as a level of the linear lattice). The number of such subspaces is denoted by the $q$-binomial coefficient $\left[\begin{array}{l}n \\ k\end{array}\right]_{q}=\prod_{0 \leqslant i<k} \frac{q^{n-i}-1}{q^{k-i}-1}$. When $k=1$, we write $[n]_{q}=\left[\begin{array}{l}n \\ 1\end{array}\right]_{q}$. Let $[n]_{q}$ ! $=\prod_{i=1}^{n}[i]_{q}$. Then, it is easy to check that

$$
\left[\begin{array}{l}
n \\
k
\end{array}\right]_{q}=\frac{[n]_{q} !}{[k]_{q} ![n-k]_{q} !} .
$$

The general study of forbidden poset problems in the linear lattice was initiated by Ghassan and Shahriari [9]. For any collection of finite posets $\mathcal{P}$, let $\operatorname{La}_{q}(n, \mathcal{P})$ be the maximum size of a family of subspaces of $V$ (viewed as a poset under inclusion) which does not contain any $P \in \mathcal{P}$ as a subposet, and let $\operatorname{La}_{q}^{*}(n, \mathcal{P})$ be the maximum size of a family of subspaces of $V$ which does not contain any $P \in \mathcal{P}$ as an induced subposet. We write simply $\operatorname{La}_{q}(n, P)$ and $\operatorname{La}_{q}^{*}(n, P)$ if $\mathcal{P}=\{P\}$ for some poset $P$. We denote by $\Sigma_{q}(n, k)$ the sum of the $k$-largest $q$-binomial coefficients of the form $\left[\begin{array}{c}n \\ i\end{array}\right]_{q}$.

Let $\mathrm{V}$ and $\Lambda$ be the posets on three elements $x, y, z$ defined by the relations $x, y>z$ and $x, y<z$, respectively. In 1983, Katona and Tarján [10] proved the following result.

Theorem 1 (Katona and Tarján [10]).

$$
\mathrm{La}(n,\{\mathrm{~V}, \Lambda\})=\mathrm{La}^{*}(n,\{\mathrm{~V}, \Lambda\})=2\left(\begin{array}{c}
n-1 \\
\left\lfloor\frac{n-1}{2}\right\rfloor
\end{array}\right) .
$$

Remark 2. In Theorem 1, an extremal construction is given by the family

$$
\left\{F: 1 \notin F,|F|=\left\lfloor\frac{n-1}{2}\right\rfloor\right\} \cup\left\{F \cup\{1\}: 1 \notin F,|F|=\left\lfloor\frac{n-1}{2}\right\rfloor\right\} .
$$

Shahriari and $\mathrm{Yu}[13]$ showed that in the linear lattice we have the following.

Theorem 3 (Shahriari and Yu [13]).

$$
\mathrm{La}_{q}(n,\{\mathrm{~V}, \Lambda\})=\left[\begin{array}{c}
n \\
\left\lfloor\frac{n}{2}\right\rfloor
\end{array}\right]_{q} .
$$

The extremal construction is either $\left[\begin{array}{c}V \\ {\left[\frac{n}{2}\right]}\end{array}\right]$ or $\left[\begin{array}{c}V \\ {\left[\frac{n}{2}\right]}\end{array}\right]$, except in the case $n=3$ and $q=2$, in which we have two other constructions shown in Figure 2. 
We prove the following induced version of Theorem 3.

\section{Theorem 4.}

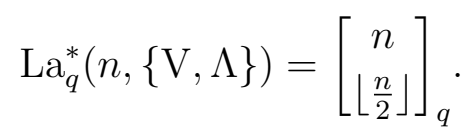

The extremal construction is either $\left[\begin{array}{c}V \\ {\left[\frac{n}{2}\right\rfloor}\end{array}\right]_{q}$ or $\left[\begin{array}{c}V \\ \left\lceil\frac{n}{2}\right\rceil\end{array}\right]_{q}$, except in the case $n=3$ and $q=2$, in which we have two other constructions shown in Figure 2.

Let $\mathrm{V}_{k}$ denote the poset with elements $x_{1}, x_{2}, \ldots, x_{k}, y$ such that $x_{1}, x_{2}, \ldots, x_{k}>y$, and let $\Lambda_{k}$ denote the same poset but with all relations reversed. In the case when $k$ or $l$ is at least 3 only asymptotic results are known for $\mathrm{La}\left(n,\left\{\mathrm{~V}_{k}, \Lambda_{l}\right\}\right)$ (see [15] and [4]).

In the linear lattice, on the other hand, one can prove exact results for larger $k$ and $l$ as well. Shahriari and $\mathrm{Yu}[13]$ proved the following.

Theorem 5 (Shahriari and $\mathrm{Yu}[13])$. Let $n$ be an even integer, and $k, l$ be two integers such that $k, l \leqslant q$. Then

$$
\operatorname{La}_{q}\left(n,\left\{\mathrm{~V}_{k}, \Lambda_{l}\right\}\right)=\left[\begin{array}{c}
n \\
\frac{n}{2}
\end{array}\right]_{q},
$$

and the only $\left\{\mathrm{V}_{k}, \Lambda_{l}\right\}$-free family of maximum size is $\left[\begin{array}{c}V \\ \frac{n}{2}\end{array}\right]_{q}$.

We extend Theorem 5 by weakening the conditions on $k$ and $l$.

Theorem 6. Let $n$ be an even integer, and $k, l$ be two integers such that $k, l \leqslant q^{\frac{n}{2}}$. Then

$$
\operatorname{La}_{q}\left(n,\left\{\mathrm{~V}_{k}, \Lambda_{l}\right\}\right)=\left[\begin{array}{c}
n \\
\frac{n}{2}
\end{array}\right]_{q},
$$

and the only $\left\{\mathrm{V}_{k}, \Lambda_{l}\right\}$-free family of maximum size is $\left[\begin{array}{c}V \\ \frac{n}{2}\end{array}\right]_{q}$.

In the induced case, we have the following two results.

Theorem 7. Let $n$ be an even integer and let $k, l$ be two integers such that $k, l \leqslant q$, then

$$
\mathrm{La}_{q}^{*}\left(n,\left\{\mathrm{~V}_{k}, \Lambda_{l}\right\}\right)=\left[\begin{array}{c}
n \\
\frac{n}{2}
\end{array}\right]_{q},
$$

and the only maximum size $\left\{\mathrm{V}_{k}, \Lambda_{l}\right\}$-free family is $\left[\begin{array}{c}V \\ \frac{n}{2}\end{array}\right]_{q}$.

Theorem 8. Let $n$ be an odd integer and let $k, l$ be two integers such that $k, l \leqslant\left(1-\frac{\sqrt{2}}{2}\right) q$, then

$$
\mathrm{La}_{q}^{*}\left(n,\left\{\mathrm{~V}_{k}, \Lambda_{l}\right\}\right)=\left[\begin{array}{c}
n \\
\frac{n-1}{2}
\end{array}\right]_{q},
$$

and any maximum size $\left\{\mathrm{V}_{k}, \Lambda_{l}\right\}$-free family is either $\left[\begin{array}{c}V \\ \frac{n-1}{2}\end{array}\right]_{q}$ or $\left[\begin{array}{c}V \\ \frac{n+1}{2}\end{array}\right]_{q}$. 
The butterfly poset, $B$, is defined by 4 elements $a, b, c, d$ with $a, b<c, d$. De Bonis, Katona and Swanepoel [5] proved the following theorem.

Theorem 9 (De Bonis, Katona and Swanepoel [5]).

$$
\mathrm{La}(n, B)=\Sigma(n, 2) .
$$

Equality occurs only for a family consisting of the union of two consecutive levels in the Boolean lattice of largest size.

We denote by $Y_{k}$ the poset with elements $x_{1}, x_{2}, \ldots, x_{k}, y, z$ such that $x_{1} \leqslant x_{2} \leqslant$ $\cdots \leqslant x_{k} \leqslant y, z$ and $Y_{k}^{\prime}$ the same poset but with all relations reversed. In the proof of Theorem 9, De Bonis, Katona and Swanepoel actually proved a stronger result by determining $\mathrm{La}\left(n,\left\{Y_{2}, Y_{2}^{\prime}\right\}\right)$. Later pairs of posets $\left\{Y_{k}, Y_{k}^{\prime}\right\}$ were investigated for their own sake. Methuku and Tompkins [12] obtained the following theorem.

Theorem 10 (Methuku and Tompkins [12]). Let $k \geqslant 2$ and $n \geqslant k+1$, then

$$
\operatorname{La}\left(n,\left\{Y_{k}, Y_{k}^{\prime}\right\}\right)=\Sigma(n, k) .
$$

Martin et al. [11] and Tompkins and Wang [16] (these results were strengthened in Gerbner et al. [7]) proved the induced version of Theorem 10 independently.

Theorem 11 (Martin et al. [11], Tompkins and Wang [16], Gerbner et al. [7]). Let $k \geqslant 2$ and $n \geqslant k+1$, then

$$
\mathrm{La}^{*}\left(n,\left\{Y_{k}, Y_{k}^{\prime}\right\}\right)=\Sigma(n, k) \text {. }
$$

In the vector space setting, Shahriari and Yu [13] proved a version of Theorem 9 holds. Namely, they proved the following.

Theorem 12 (Shahriari and $\mathrm{Yu}[13])$. Let $n \geqslant 3$ be an integer and $q$ be a power of a prime, then

$$
\operatorname{La}_{q}(n, B)=\operatorname{La}_{q}\left(n,\left\{Y_{2}, Y_{2}^{\prime}\right\}\right)=\Sigma_{q}(n, 2) .
$$

Equality occurs only for a family consisting of the union of two consecutive levels in the linear lattice of maximum size.

Furthermore, they posed a conjecture for the case when $\left\{Y_{k}, Y_{k}^{\prime}\right\}$ is forbidden.

For any poset $P$, let $|P|$ be the size of $P$ and $h(P)$ be the length of the largest chain in $P$. Burcsi and Nagy [1] and Grósz, Methuku and Tompkins [8] proved the following theorems for any poset $P$ (another result in this direction was obtain by Chen and Li [2]).

Theorem 13 (Burcsi and Nagy [1]). For any poset $P$, when $n$ is sufficiently large, we have

$$
\mathrm{La}(n, P) \leqslant\left(\frac{|P|+h(P)}{2}-1\right)\left(\begin{array}{c}
n \\
\left\lfloor\frac{n}{2}\right\rfloor
\end{array}\right) .
$$


Theorem 14 (Grósz, Methuku and Tompkins [8]). For any poset $P$, when $n$ is sufficiently large, we have

$$
\mathrm{La}(n, P) \leqslant \frac{1}{2^{k-1}}\left(|P|+(3 k-5) 2^{k-2}(h(P)-1)-1\right)\left(\begin{array}{c}
n \\
\left\lfloor\frac{n}{2}\right\rfloor
\end{array}\right),
$$

for any fixed $k$.

We will prove that a version of these theorems holds in the vector space case as well.

The rest of this paper is organized as follows. In the next section, we present some preliminary results. Then we will prove Theorems 4, 7 and 8 in Section 3. In the last section, we prove a general LYM-type lemma and use this lemma to prove the vector space analogues of Theorems 10,11, 13 and 14. We note that a recent manuscript of Gerbner [6] independently initiates a general study of LYM-type properties of the linear lattice and implies some similar results.

\section{Preliminary results}

In this section, let $\mathcal{F}$ be a $\left\{\mathrm{V}_{k}, \Lambda_{l}\right\}$-free family of subspaces of $V$, and let $\mathcal{F}_{s}=\mathcal{F} \cap\left[\begin{array}{l}V \\ s\end{array}\right]_{q}$. Now, we define the bipartite graph $\left(\mathcal{F}_{s} \cup\left(\left[\begin{array}{c}V \\ s-1\end{array}\right]_{q} \backslash \mathcal{F}_{s-1}\right), E\right)$, where

$$
E=\left\{\left(A \in \mathcal{F}_{s}, B \in\left(\left[\begin{array}{c}
V \\
s-1
\end{array}\right]_{q} \backslash \mathcal{F}_{s-1}\right)\right): B \subset A\right\} .
$$

Let $\mathcal{F}_{s}^{\prime}$ be any subset of $\mathcal{F}_{s}$, and

$$
N_{s-1}\left(\mathcal{F}_{s}^{\prime}\right)=\left\{B \in\left(\left[\begin{array}{c}
V \\
s-1
\end{array}\right]_{q} \backslash \mathcal{F}_{s-1}\right):(A, B) \in E \text { for some } A \in \mathcal{F}_{s}^{\prime}\right\} .
$$

Before beginning the proof, we need some preliminary results. Lemma 15 and Corollary 16 are motivated by an idea from [10].

Lemma 15. Let $n$ be an even integer, and let $k, l$ be two integers such that $k, l \leqslant q^{\frac{n}{2}}$. Then, $\operatorname{La}_{q}\left(n,\left\{\mathrm{~V}_{k}, \Lambda_{l}\right\}\right)$ can be realized with a family $\mathcal{G}$ of subspaces $G$ satisfying $\operatorname{dim}(G) \leqslant$ $\frac{n}{2}$.

Proof: We first prove that for $s \geqslant \frac{n}{2}+1$, the bipartite graph $\left(\mathcal{F}_{s} \cup\left(\left[\begin{array}{c}V \\ s-1\end{array}\right] \backslash \mathcal{F}_{s-1}\right), E\right)$ contains a matching such that every element of $\mathcal{F}_{s}$ is contained in some edge. To prove this, it is enough to check the condition of Hall's theorem, that is

$$
\left|N_{s-1}\left(\mathcal{F}_{s}^{\prime}\right)\right| \geqslant\left|\mathcal{F}_{s}^{\prime}\right|
$$

for any $\mathcal{F}_{s}^{\prime} \subseteq \mathcal{F}_{s}$.

Since $\mathcal{F}$ is $\Lambda_{l}$-free, every $s$-dimensional subspace in $\mathcal{F}_{s}^{\prime}$ has at most $(l-1)$ subspaces in $\mathcal{F}_{s-1}$. Hence, every $s$-dimensional subspace in $\mathcal{F}_{s}^{\prime}$ has at least $[s]_{q}-l+1$ subspaces 
in $N_{s-1}\left(\mathcal{F}_{s}^{\prime}\right)$. On the other hand, every $(s-1)$-dimensional subspace in $N_{s-1}\left(\mathcal{F}_{s}^{\prime}\right)$ has at most $[n-s+1]_{q}$ superspaces in $\mathcal{F}_{s}^{\prime}$. We have

$$
\begin{aligned}
\frac{\left|N_{s-1}\left(\mathcal{F}_{s}^{\prime}\right)\right|}{\left|\mathcal{F}_{s}^{\prime}\right|} & \geqslant \frac{[s]_{q}-l+1}{[n-s+1]_{q}} \geqslant \frac{\frac{q^{s}-1}{q-1}-l+1}{\frac{q^{n-s+1}-1}{q-1}} \\
& \geqslant \frac{q^{\frac{n}{2}+1}-1-\left(q^{\frac{n}{2}}-1\right)(q-1)}{q^{\frac{n}{2}}-1} \\
& \geqslant \frac{q^{\frac{n}{2}}+q-2}{q^{\frac{n}{2}}-1} \geqslant 1,
\end{aligned}
$$

since $s \geqslant \frac{n}{2}+1, l \leqslant q^{\frac{n}{2}}$ and $q \geqslant 2$. Applying Hall's theorem, let $M$ be a matching which saturates every vertex in $\mathcal{F}_{s}$, and let $\mathcal{F}_{s-1}^{*}$ be the set of neighbors of $\mathcal{F}_{s}$ contained in edges of $M$. Clearly, $\mathcal{F}_{s-1}^{*} \cap \mathcal{F}_{s-1}=\emptyset$, and $\left|\mathcal{F}_{s-1}^{*}\right|=\left|\mathcal{F}_{s}\right|$.

Now, let $t=t(\mathcal{F})$ be the largest integer $s$ satisfying $\mathcal{F}_{s} \neq \emptyset$ in the family $\mathcal{F}$. We iteratively replace $\mathcal{F}$ with $\left(\mathcal{F} \backslash \mathcal{F}_{t}\right) \cup \mathcal{F}_{t-1}^{*}$ until $t \leqslant \frac{n}{2}$. Call the resulting family $\mathcal{G}$. Clearly, $|\mathcal{G}|=|\mathcal{F}|$, and $\mathcal{G}$ is $\left\{\mathrm{V}_{k}, \Lambda_{l}\right\}$-free since $\mathcal{F}$ is $\left\{\mathrm{V}_{k}, \Lambda_{l}\right\}$-free.

Since linear lattices are symmetric, one can use the same idea to prove the following corollary.

Corollary 16. Let $n$ be an even integer, and $k, l$ be two integers such that $k, l \leqslant q^{\frac{n}{2}}$. Then, $\operatorname{La}_{q}\left(n,\left\{\mathrm{~V}_{k}, \Lambda_{l}\right\}\right)$ can be realized with a family $\mathcal{G}$ of subspaces $G$ satisfying $\operatorname{dim}(G) \geqslant \frac{n}{2}$.

The next technical lemma will be needed for determining the structure of the extremal families.

Lemma 17. Let $\mathcal{F}$ be a family such that $\operatorname{dim}(F)=\left\lceil\frac{n}{2}\right\rceil$ or $\left\lceil\frac{n}{2}\right\rceil+1$ for every $F \in \mathcal{F}$. If $|\mathcal{F}|=\left[\begin{array}{c}n \\ \left\lceil\frac{n}{2}\right\rceil\end{array}\right]_{q}$ and $\mathcal{F}_{\left\lceil\frac{n}{2}\right\rceil+1} \neq \emptyset$, then $\mathcal{F}$ contains a copy of $\Lambda_{q\left\lceil\frac{n}{2}\right\rceil}$.

Proof: Any $F \in \mathcal{F}_{\left\lceil\frac{n}{2}\right\rceil+1}$ has $\left[\left\lceil\frac{n}{2}\right\rceil+1\right]_{q}$ subspaces in $\left[\begin{array}{c}V \\ \left\lceil\frac{n}{2}\right\rceil\end{array}\right]$, and any $F^{\prime} \in\left[\begin{array}{c}V \\ \left\lceil\frac{n}{2}\right\rceil\end{array}\right]_{q}$ has $\left[\left\lfloor\frac{n}{2}\right\rfloor\right]_{q}$ superspaces in $\left[\begin{array}{c}V \\ \left\lceil\frac{n}{2}\right\rceil+1\end{array}\right]_{q}$. We may now show by a simple averaging argument that there exists an $F \in \mathcal{F}_{\left\lceil\frac{n}{2}\right\rceil+1}$ such that $F$ has at least $q^{\left\lceil\frac{n}{2}\right\rceil}$ subspaces in $\mathcal{F}_{\left\lceil\frac{n}{2}\right\rceil}$. Indeed, by the assumption that $|\mathcal{F}|=\left[\begin{array}{c}n \\ \left\lceil\frac{n}{2}\right\rceil\end{array}\right]$, the number of relations between $\mathcal{F}_{\left\lceil\frac{n}{2}\right\rceil+1}$ and $\mathcal{F}_{\left\lceil\frac{n}{2}\right\rceil}$ is at least

$$
\begin{aligned}
& {\left[\left\lceil\frac{n}{2}\right\rceil+1\right]_{q}\left|\mathcal{F}_{\left\lceil\frac{n}{2}\right\rceil+1}\right|-\left[\left\lfloor\frac{n}{2}\right\rfloor\right]_{q}\left|\left[\begin{array}{c}
V \\
\left\lceil\frac{n}{2}\right\rceil
\end{array}\right]_{q} \backslash \mathcal{F}_{\left\lceil\frac{n}{2}\right\rceil}\right|} \\
& =\left|\mathcal{F}_{\left\lceil\frac{n}{2}\right\rceil+1}\right|\left(\left[\left\lceil\frac{n}{2}\right\rceil+1\right]_{q}-\left[\left\lfloor\frac{n}{2}\right\rfloor\right]_{q}\right) \\
& =\left|\mathcal{F}_{\left\lceil\frac{n}{2}\right\rceil+1}\right| \frac{q^{\left\lceil\frac{n}{2}\right\rceil+1}-q^{\left\lfloor\frac{n}{2}\right\rfloor}}{q-1} \\
& \geqslant\left|\mathcal{F}_{\left\lceil\frac{n}{2}\right\rceil+1}\right| q^{\left\lceil\frac{n}{2}\right\rceil} .
\end{aligned}
$$

Thus, on average an element of $\mathcal{F}_{\left\lceil\frac{n}{2}\right\rceil+1}$ contains at least $q^{\left\lceil\frac{n}{2}\right\rceil}$ subspaces in $\mathcal{F}_{\left\lceil\frac{n}{2}\right\rceil}$.

In the same way one can show the following. 
Lemma 18. Let $\mathcal{F}$ be a family such that $\operatorname{dim}(F)=\left\lfloor\frac{n}{2}\right\rfloor$ or $\left\lfloor\frac{n}{2}\right\rfloor-1$ for every $F \in \mathcal{F}$. If $|\mathcal{F}|=\left[\begin{array}{c}n \\ \left\lfloor\frac{n}{2}\right\rfloor\end{array}\right]_{q}$ and $\mathcal{F}_{\left\lfloor\frac{n}{2}\right\rfloor-1} \neq \emptyset$, then $\mathcal{F}$ contains a copy of $\mathrm{V}_{q^{\left\lceil\frac{n}{2}\right\rceil}}$.

Now, we can prove Theorem 6.

Proof: Combining Lemma 15 and Corollary 16, it is easy to see that

$$
\operatorname{La}_{q}\left(n,\left\{\mathrm{~V}_{k}, \Lambda_{l}\right\}\right)=\left[\begin{array}{c}
n \\
\frac{n}{2}
\end{array}\right]_{q}
$$

Now we prove that if $\mathcal{F}$ is a $\left\{\mathrm{V}_{k}, \Lambda_{l}\right\}$-free family of maximum size, then $\mathcal{F}=\left[\begin{array}{c}V \\ \frac{n}{2}\end{array}\right]$. Suppose not. If there is a subspace $F \in \mathcal{F}$ of dimension larger than $\frac{n}{2}$, then we may assume, without loss of generality, that $|\mathcal{F}|=\left[\begin{array}{c}n \\ \frac{n}{2}\end{array}\right]_{q}$ and for every $F \in \mathcal{F}, \frac{n}{2} \leqslant \operatorname{dim}(F) \leqslant$ $\frac{n}{2}+1$ and $\mathcal{F}_{\frac{n}{2}+1} \neq \emptyset$. By Lemma $17, \mathcal{F}$ contains a copy of $\Lambda_{l}$, a contradiction. The case when $\mathcal{F}^{2}$ contains only subspaces of dimension at most $\frac{n}{2}$ is handled similarly by Lemma 18.

\section{Proofs of Theorems 4,7 and 8}

In this section, let $\mathcal{F}$ be an induced $\left\{\mathrm{V}_{k}, \Lambda_{l}\right\}$-free family, and let $\mathcal{F}_{s}=\left[\begin{array}{l}V \\ s\end{array}\right]_{q} \cap \mathcal{F}$. We call a subspace $F \in \mathcal{F}$ small if for any other $F^{\prime} \in \mathcal{F}, F^{\prime} \nsubseteq F$. For every $A \in \mathcal{F}_{s}$, let $F_{1}, F_{2}, \ldots, F_{r}$ be $r$ small proper subspaces of $A$ in $\mathcal{F}$. We note that the $F_{i}$ 's enumerate all of the proper small subspaces of $A$. Clearly, $0 \leqslant r \leqslant l-1$ since $\mathcal{F}$ is induced $\Lambda_{l}$-free $(r=0$ if $A$ is small). Let $f_{1} \subseteq F_{1}, f_{2} \subseteq F_{2}, \ldots, f_{r} \subseteq F_{r}$ be $r$ one dimensional subspaces (note that $f_{1}, f_{2}, \ldots, f_{r}$ are not necessarily distinct). Then, we have the following proposition.

Proposition 19. If $F$ is a subspace of $A$ and $F \in \mathcal{F}$, then $f_{i} \subseteq F$ for some $i \in[r]$.

Proof: The subspace $F$ is either small (suppose $F=F_{i}$ in this case) or contains some small subspace $F_{i}$. In both cases we have $f_{i} \subseteq F_{i} \subseteq F$.

Now, we define a family $M(A)$ collecting all $(s-1)$-subspaces of $A$ which do not contain any of the $f_{i}$.

$$
M(A)=\left\{B: \operatorname{dim}(B)=s-1, f_{1} \nsubseteq \subseteq B, f_{2} \not \subset B, \cdots, f_{r} \nsubseteq B \text { and } B \subseteq A\right\} .
$$

By Proposition 19, we have that the following properties of $M(A)$ hold.

Proposition 20. (i) For any $B \in M(A)$ and $F \in \mathcal{F}$ such that $\operatorname{dim}(F) \leqslant s-2, F \nsubseteq B$.

(ii) $M(A) \cap \mathcal{F}_{s-1}=\emptyset$.

(iii) $|M(A)| \geqslant[s]_{q}-(l-1)[s-1]_{q}$.

Proof: (i) If $F \nsubseteq A$, then $F \nsubseteq B$ since $B \subseteq A$. Let $F \subseteq A$, then by Proposition 19, $f_{i} \subseteq F$ for some $i \in[r]$. However, by the definition of $M(A), f_{i} \nsubseteq B$, and so $F \nsubseteq B$. 
(ii) Suppose not. Let $B \in M(A) \cap \mathcal{F}_{s-1}$. We have that $B$ contains a one dimensional subspace $f_{i}$ by Proposition 19 , but $f_{i} \not \subset B$ by the definition of $M(A)$, a contradiction.

(iii) For an $s$-dimensional subspace $A$, there are $[s]_{q}(s-1)$-dimensional subspaces of $A$. At most $[s-1]_{q}$ among them contain $f_{i}$ for each $f_{i}$. So

$$
|M(A)| \geqslant[s]_{q}-r[s-1]_{q} \geqslant[s]_{q}-(l-1)[s-1]_{q},
$$

as required.

Now, we define the bipartite graph $\left(\mathcal{F}_{s} \cup\left(\left[\begin{array}{c}V \\ { }_{s-1}\end{array}\right]_{q} \backslash \mathcal{F}_{s-1}\right), E\right)$, where

$$
E=\left\{(A, B): A \in \mathcal{F}_{s}, B \in M(A)\right\} .
$$

Let $\mathcal{F}_{s}^{\prime}$ be any subset of $\mathcal{F}_{s}$, and

$$
N_{s-1}\left(\mathcal{F}_{s}^{\prime}\right)=\left\{B:(A, B) \in E \text { for some } A \in \mathcal{F}_{s}^{\prime}\right\} .
$$

Lemma 21. Let $n$ be an odd integer, and $k, l$ be two integers such that $k, l \leqslant q$. Then, $\mathrm{La}_{q}^{*}\left(n,\left\{\mathrm{~V}_{k}, \Lambda_{l}\right\}\right)$ can be realized with a family $\mathcal{G}$ of subspaces $G$ satisfying $\operatorname{dim}(G) \leqslant \frac{n+1}{2}$.

Proof: We first show that for $s \geqslant \frac{n+3}{2}$, the bipartite graph $\left(\mathcal{F}_{s} \cup\left(\left[\begin{array}{c}V \\ s-1\end{array}\right]_{q} \backslash \mathcal{F}_{s-1}\right), E\right)$ contains a matching such that every element of $\mathcal{F}_{s}$ is contained in some edge. By Hall's theorem, it is enough to prove

$$
\left|N_{s-1}\left(\mathcal{F}_{s}^{\prime}\right)\right| \geqslant\left|\mathcal{F}_{s}^{\prime}\right|
$$

for any $\mathcal{F}_{s}^{\prime} \subseteq \mathcal{F}_{s}$.

On the one hand, by (ii) from Proposition 20, $M(A) \cap \mathcal{F}_{s-1}=\emptyset$, then every $s$ dimensional subspace $A$ in $\mathcal{F}_{s}^{\prime}$ has $|M(A)|$ subspaces in $N_{s-1}\left(\mathcal{F}_{s}^{\prime}\right)$. On the other hand, every $(s-1)$-dimensional subspace in $N_{s-1}\left(\mathcal{F}_{s}^{\prime}\right)$ has at most $[n-s+1]_{q}$ superspaces in $\mathcal{F}_{s}^{\prime}$. Then, by (iii) from Proposition 20, we have

$$
\begin{aligned}
\frac{\left|N_{s-1}\left(\mathcal{F}_{s}^{\prime}\right)\right|}{\left|\mathcal{F}_{s}^{\prime}\right|} \geqslant \frac{[s]_{q}-(l-1)[s-1]_{q}}{[n-s+1]_{q}} \geqslant \frac{\frac{q^{s}-1}{q-1}-\frac{(l-1)\left(q^{s-1}-1\right)}{q-1}}{\frac{q^{n-s+1}-1}{q-1}} \\
\geqslant \frac{q^{s-1}+q-2}{q^{n-s+1}-1} \geqslant \frac{q^{\frac{n+1}{2}}+q-2}{q^{\frac{n-1}{2}}-1} \geqslant 1,
\end{aligned}
$$

since $s \geqslant \frac{n+3}{2}, l \leqslant q$ and $q \geqslant 2$. Let $M$ be a matching which saturates every vertex in $\mathcal{F}_{s}$, and $\mathcal{F}_{s-1}^{*}$ be matched under $M$. Clearly, $\left|\mathcal{F}_{s-1}^{*}\right|=\left|\mathcal{F}_{s}\right|$, and $\mathcal{F}_{s-1}^{*} \cap \mathcal{F}_{s-1}=\emptyset$ by (ii) from Proposition 20. Now, let $t=t(\mathcal{F})$ be the largest integer $s$ satisfying $\mathcal{F}_{s} \neq \emptyset$ in our family $\mathcal{F}$. We repeatedly replace $\mathcal{F}$ by $\left(\mathcal{F} \backslash \mathcal{F}_{t}\right) \cup \mathcal{F}_{t-1}^{*}$ until $t \leqslant \frac{n+1}{2}$. Call the resulting family $\mathcal{G}$. Clearly, $|\mathcal{G}|=|\mathcal{F}|$. Then it is enough to show that $\mathcal{F}$ is induced $\left\{\mathrm{V}_{k}, \Lambda_{l}\right\}$-free in every step.

By contradiction, assume that at some step $\mathcal{F}$ is $\left\{\mathrm{V}_{k}, \Lambda_{l}\right\}$-free but $\left(\mathcal{F} \backslash \mathcal{F}_{t}\right) \cup \mathcal{F}_{t-1}^{*}$ contains $\mathrm{V}_{k}$ or $\Lambda_{l}$. We distinguish two cases.

Case 3.1. $\left(\mathcal{F} \backslash \mathcal{F}_{t}\right) \cup \mathcal{F}_{t-1}^{*}$ contains an induced $\Lambda_{l}$. 
Let $F_{1}, F_{2}, F_{3}, \ldots, F_{l} \subset F$ be $l+1$ subspaces in $\left(\mathcal{F} \backslash \mathcal{F}_{t}\right) \cup \mathcal{F}_{t-1}^{*}$ which form an induced $\Lambda_{l}$. Then, $F \in \mathcal{F}_{t-1}^{*}$, since $\mathcal{F}$ is induced $\Lambda_{l}$-free. Let $A$ be matched with $F$ under $M$, then $A$ together with $F_{1}, F_{2}, F_{3}, \ldots, F_{l}$ form an induced $\Lambda_{l}$ in $\mathcal{F}$, a contradiction.

Case 3.2. $\left(\mathcal{F} \backslash \mathcal{F}_{t}\right) \cup \mathcal{F}_{t-1}^{*}$ contains an induced $\mathrm{V}_{k}$.

Let $F \subset F_{1}, F_{2}, F_{3}, \ldots, F_{k}$ be $k+1$ subspaces in $\left(\mathcal{F} \backslash \mathcal{F}_{t}\right) \cup \mathcal{F}_{t-1}^{*}$ which form an induced $\mathrm{V}_{k}$. Since $\mathcal{F}$ is induced $\mathrm{V}_{k}$-free, we may suppose $F_{1} \in \mathcal{F}_{t-1}^{*}$. Let $A$ be matched with $F_{1}$ under $M$. Then $F_{1} \in M(A)$. Note that $F \in \mathcal{F}$ and $\operatorname{dim}(F) \leqslant t-2$. By (i) from Proposition 20, $F \nsubseteq F_{1}$, a contradiction.

Using the same ideas from Section 2, one can prove the following corollaries similarly.

Corollary 22. Let $n$ be an odd integer, and $k, l$ be two integers such that $k, l \leqslant q$. Then, $\operatorname{La}_{q}^{*}\left(n,\left\{\mathrm{~V}_{k}, \Lambda_{l}\right\}\right)$ can be realized with a family $\mathcal{G}$ of subspaces $G$ satisfying $\frac{n-1}{2} \leqslant \operatorname{dim}(G) \leqslant$ $\frac{n+1}{2}$.

Corollary 23. Let $n$ be an even integer, and $k, l$ be two integers such that $k, l \leqslant q$. Then, $\operatorname{La}_{q}^{*}\left(n,\left\{\mathrm{~V}_{k}, \Lambda_{l}\right\}\right)$ can be realized with a family $\mathcal{G}$ of subspaces $G$ satisfying $\operatorname{dim}(G)=\frac{n}{2}$.

Theorem 7 follows from Corollary 23 and the equality cases are again settled by applying Lemmas 17 and 18. (Once a family is contained in two levels there is no distinction between an induced and noninduced copy of $\mathrm{V}_{k}$ or $\Lambda_{l}$.)

Now, we turn to prove Theorem 8. Before beginning the proof, we need the following lemma.

Lemma 24. Let $V_{3}$ be a 3-dimensional vector space over $\mathbb{F}_{q}$. If $\mathcal{F} \subseteq\left(\left[\begin{array}{c}V_{3} \\ 1\end{array}\right]_{q} \cup\left[\begin{array}{c}V_{3} \\ 2\end{array}\right]_{q}\right)$ is $\left\{V_{k}, \Lambda_{l}\right\}$-free, where $k, l \leqslant q-\frac{\sqrt{2}}{2} q$, then

$$
|\mathcal{F}| \leqslant q^{2}+q+1
$$

and the only families which attain equality are $\left[\begin{array}{c}V_{3} \\ 1\end{array}\right]_{q}$ and $\left[\begin{array}{c}V_{3} \\ 2\end{array}\right]_{q}$.

Proof: $\quad$ Let $\mathcal{F}=\mathcal{A} \cup \mathcal{B}$, where $\mathcal{A} \subseteq\left[\begin{array}{c}V_{3} \\ 2\end{array}\right]_{q}$ and $\mathcal{B} \subseteq\left[\begin{array}{c}V_{3} \\ 1\end{array}\right]_{q}$, and let $\mathcal{A}^{\prime}=\left[\begin{array}{c}V_{3} \\ 2\end{array}\right]_{q} \backslash \mathcal{A}$ and $\mathcal{B}^{\prime}=\left[\begin{array}{c}V_{3} \\ 1\end{array}\right]_{q} \backslash \mathcal{B}$.

We prove the inequality by contradiction. Suppose that $|\mathcal{F}|=|\mathcal{A}|+|\mathcal{B}| \geqslant q^{2}+q+2$. Note that $\left|\left[\begin{array}{c}V_{3} \\ 1\end{array}\right]_{q}\right|=\left|\left[\begin{array}{c}V_{3} \\ 2\end{array}\right]_{q}\right|=q^{2}+q+1$, so we have $|\mathcal{A}|>\left|\mathcal{B}^{\prime}\right|$ and $|\mathcal{B}|>\left|\mathcal{A}^{\prime}\right|$. Since $\mathcal{F}$ is $\Lambda_{l}$-free, for every $A \in \mathcal{A}$, the number of subspaces of $A$ in $\mathcal{B}$ is at most $l-1$, thus the number of subspaces of $A$ in $\mathcal{B}^{\prime}$ is at least $(q+1)-(l-1)=q+2-l$. Since $|\mathcal{A}|>\left|\mathcal{B}^{\prime}\right|$, there exists a subspace $B \in \mathcal{B}^{\prime}$ with at least $q+3-l$ superspaces $A_{1}, A_{2}, \ldots, A_{q+3-l}$ in $\mathcal{A}$ by the pigeonhole principle.

For $1 \leqslant i, j \leqslant q+3-l, A_{i}$ and $A_{j}$ have only one common subspace $B$, since there is no butterfly in two consecutive levels of a linear lattice. So we have

$$
\left|\mathcal{B}^{\prime}\right| \geqslant(q+3-l)(q+1-l)+1,
$$

and similarly, we have

$$
|\mathcal{B}|>\left|\mathcal{A}^{\prime}\right| \geqslant(q+3-k)(q+1-k)+1
$$




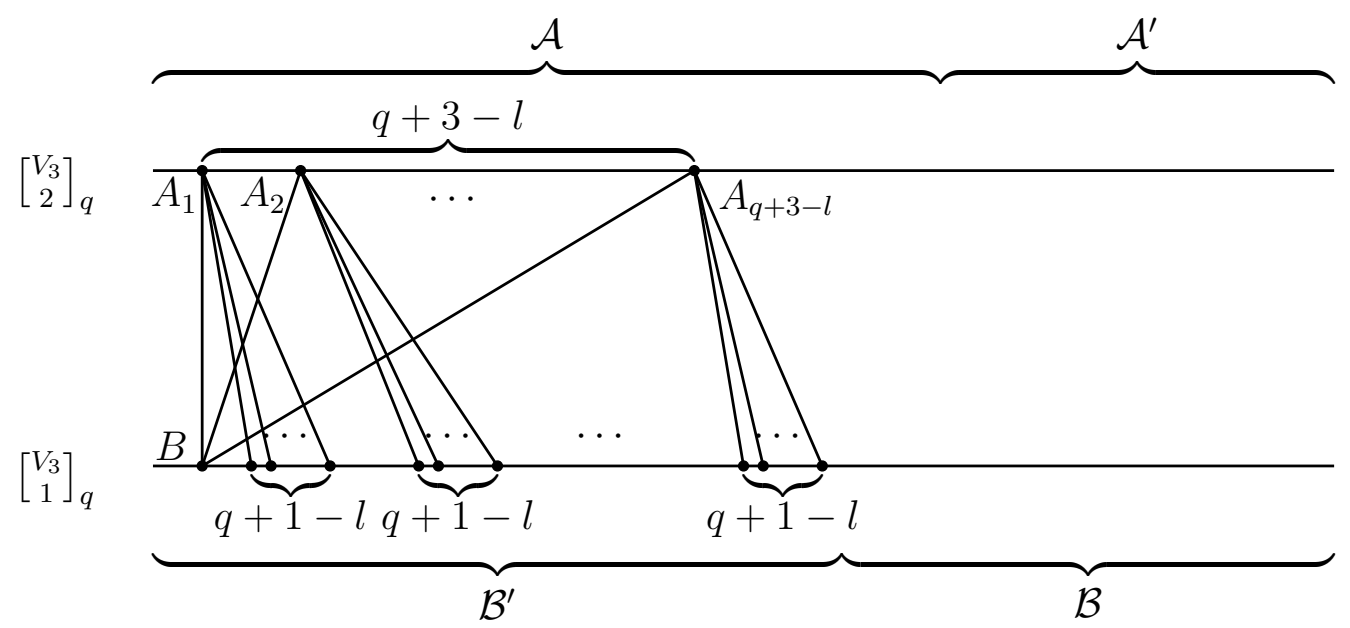

Figure 1: $\left[\begin{array}{c}V_{3} \\ 1\end{array}\right]_{q} \cup\left[\begin{array}{c}V_{3} \\ 2\end{array}\right]_{q}$.

since $\mathcal{F}$ is $\mathrm{V}_{k}$-free. Then,

$$
q^{2}+q+1=\left|\left[\begin{array}{l}
V \\
1
\end{array}\right]_{q}\right|=\left|\mathcal{B}^{\prime}\right|+|\mathcal{B}|>(q+3-l)(q+1-l)+(q+3-k)(q+1-k)+2,
$$

a contradiction when $k, l \leqslant q-\frac{\sqrt{2}}{2} q$. This completes the proof of the inequality. Furthermore, if $|\mathcal{F}|=q^{2}+q+1$ and $\mathcal{A}, \mathcal{B} \neq \emptyset$, we will have $|\mathcal{A}|=\left|\mathcal{B}^{\prime}\right|$ instead of $|\mathcal{A}|>\left|\mathcal{B}^{\prime}\right|$. Then there exists a subspace $B \in \mathcal{B}^{\prime}$ with at least $q+2-l$ superspaces in $\mathcal{A}$, and so

$$
q^{2}+q+1=|\mathcal{F}| \geqslant(q+2-l)(q+1-l)+(q+2-k)(q+1-k)+2,
$$

but this contradicts the condition $k, l \leqslant q-\frac{\sqrt{2}}{2} q$.

Remark 25. In Lemma 24, the upper bound of $|\mathcal{F}|$ is true when the weaker condition $q^{2}+q+1<(q+3-l)(q+1-l)+(q+3-k)(q+1-k)+2$ holds, and the extremal structure of $\mathcal{F}$ holds when the weaker condition $q^{2}+q+1<(q+2-l)(q+1-l)+(q+2-k)(q+1-k)+2$ is satisfied.

Now, we are ready to prove Theorem 8.

Proof of Theorem 8: A maximal chain in a linear lattice of dimension $n$ is a sequence of subspaces $V_{0}, V_{1}, \ldots, V_{n}$ where $\{0\}=V_{0} \subset V_{1} \subset \cdots \subset V_{n}=V$. We denote by $\mathcal{C}$ the set of all maximal chains in a linear lattice. Now, we double count the number of pairs $(F, C)$, where $F \in \mathcal{F}, C \in \mathcal{C}$ such that $F$ is in the chain $C$.

For every $F \in \mathcal{F}$, there are $[\operatorname{dim}(F)]_{q} ![n-\operatorname{dim}(F)]_{q}$ ! maximal chains though $F$. On the other hand, by Corollary 22, we may assume that for every $F \in \mathcal{F},(n-1) / 2 \leqslant \operatorname{dim}(F) \leqslant$ $(n+1) / 2$. Then, we consider a pair of subspaces $\left(G_{1}, G_{2}\right)$ such that $\operatorname{dim}\left(G_{1}\right)=\frac{n+3}{2}$, $\operatorname{dim}\left(G_{2}\right)=\frac{n-3}{2}$ and $G_{2} \subseteq G_{1}$. The subfamily of $\mathcal{F}$ between $G_{1}$ and $G_{2}$ satisfies the condition of Lemma 24. Hence, the size of the subfamily can be bounded as $q^{2}+q+1$, and the number of chains between $G_{1}$ and $G_{2}$ though some $F$ in the subfamily is $\left(q^{2}+\right.$ 
$q+1)(q+1)$. Clearly, the number of maximal chains between $\{0\}$ and $G_{2}\left(G_{1}\right.$ and $\left.V\right)$ is $\left[\frac{n-3}{2}\right]_{q}$ !, and the number of such pairs $\left(G_{1}, G_{2}\right)$ is $\left[\begin{array}{c}n \\ \frac{n+3}{2}\end{array}\right]_{q}\left[\frac{n+3}{2} \frac{n-3}{2}\right]$. Then, we have

$$
\sum_{F \in \mathcal{F}}[\operatorname{dim}(F)]_{q} ![n-\operatorname{dim}(F)]_{q} ! \leqslant\left[\begin{array}{c}
n \\
\frac{n+3}{2}
\end{array}\right]_{q}\left[\begin{array}{c}
\frac{n+3}{2} \\
\frac{n-3}{2}
\end{array}\right]_{q}\left(\left[\frac{n-3}{2}\right]_{q} !\right)^{2}\left(q^{2}+q+1\right)(q+1)=[n]_{q} !
$$

It follows that

$$
\frac{|\mathcal{F}|}{\left[\frac{n}{2}\right]_{q}} \leqslant \sum_{F \in \mathcal{F}} \frac{1}{\left[\begin{array}{c}
n \\
\operatorname{dim}(F)]_{q}
\end{array}\right.} \leqslant 1
$$

hence,

$$
|\mathcal{F}| \leqslant\left[\begin{array}{c}
n \\
\frac{n+1}{2}
\end{array}\right]_{q}
$$

This completes the proof of $\operatorname{La}_{q}^{*}\left(n,\left\{\mathrm{~V}_{k}, \Lambda_{l}\right\}\right)=\left[\begin{array}{c}n \\ \frac{n+1}{2}\end{array}\right]_{q}$. Since equality must hold in the first inequality of (1) when $|\mathcal{F}|=\left[\begin{array}{c}n \\ \frac{n+1}{2}\end{array}\right]_{q}$, we have the following.

Fact 26. If $\frac{n-1}{2} \leqslant \operatorname{dim}(F) \leqslant \frac{n+1}{2}$ for every $F \in \mathcal{F}$ and $|\mathcal{F}|=\left[\begin{array}{c}n \\ n+1\end{array}\right]_{q}$, then the size of the subfamily between $G_{1}$ and $G_{2}$ is $q^{2}+q+1$ for any pair $\left(G_{1}, G_{2}\right)$ such that $\operatorname{dim}\left(G_{1}\right)=\frac{n+3}{2}$, $\operatorname{dim}\left(G_{2}\right)=\frac{n-3}{2}$ and $G_{2} \subseteq G_{1}$.

We will also make use of the following simple lemma.

Lemma 27. Let $G$ be a connected, regular, bipartite graph with parts $A$ and $B$. If for some $A^{\prime} \subset A$ we have $\left|N\left(A^{\prime}\right)\right|=|A|$, then either $A^{\prime}=\emptyset$ or $A^{\prime}=A$.

Now, we show the largest induced $\left\{\mathrm{V}_{k}, \Lambda_{l}\right\}$-free family is either $\left[\begin{array}{c}V \\ \frac{n+1}{2}\end{array}\right]_{q}$ or $\left[\frac{{ }_{n-1}}{2}\right]_{q}$ by considering three cases. Assume $\mathcal{F}$ is an induced $\left\{\mathrm{V}_{k}, \Lambda_{l}\right\}$-free family of size $\left[\begin{array}{c}n \\ \frac{n+1}{2}\end{array}\right]_{q}$.

Case 3.3. For all $F \in \mathcal{F}, \operatorname{dim}(F) \geqslant \frac{n+1}{2}$.

We will show that $\mathcal{F}$ contains only subspaces of dimension $\frac{n+1}{2}$. Suppose not, then we may find an induced $\left\{\mathrm{V}_{k}, \Lambda_{l}\right\}$-free family $\mathcal{F}^{\prime}$ of size $\left[\begin{array}{c}n \\ \frac{n+1}{2}\end{array}\right]_{q}$ containing only subspaces of dimension $\frac{n+1}{2}$ and $\frac{n+3}{2}$ such that $\mathcal{F}_{\frac{n+3}{2}}^{\prime} \neq \emptyset$. However, by Lemma $17, \mathcal{F}$ contains a copy of $\Lambda_{l}$, since $l \leqslant q-\frac{\sqrt{2}}{2} q \leqslant q^{\frac{n+1}{2}}$, a contradiction. The following case can be proved by a similar argument.

Case 3.4. For all $F \in \mathcal{F}, \operatorname{dim}(F) \leqslant \frac{n-1}{2}$.

Case 3.5. There exist two subspaces $F_{1}$ and $F_{2}$ in $\mathcal{F}$ such that $\operatorname{dim}\left(F_{1}\right) \geqslant \frac{n+1}{2}$ and $\operatorname{dim}\left(F_{2}\right) \leqslant \frac{n-1}{2}$.

We will show this case is impossible. By Corollary 22 , there is an induced $\left\{\mathrm{V}_{k}, \Lambda_{l}\right\}$ free family $\mathcal{F}^{\prime}$ of the same size as $\mathcal{F}$ such that $\frac{n-1}{2} \leqslant \operatorname{dim}\left(F^{\prime}\right) \leqslant \frac{n+1}{2}$ for every $F^{\prime} \in \mathcal{F}^{\prime}$. 
Clearly, $\left|\mathcal{F}_{\frac{n+1}{2}}^{\prime}\right|+\left|\mathcal{F}_{\frac{n-1}{2}}^{\prime}\right|=\left[\begin{array}{c}n \\ \frac{n-1}{2}\end{array}\right]_{q}$. Then, by the assumption on the dimensions of $F_{1}$ and $F_{2}$, we have $\mathcal{F}_{\frac{n+1}{2}}^{\prime} \neq \emptyset$ and $\mathcal{F}_{\frac{n-1}{2}}^{\prime} \neq \emptyset$.

Let $N\left(\mathcal{F}_{\frac{n+1}{2}}^{\prime}\right)$ be the collection of subspaces of dimension $\frac{n-1}{2}$ contained in subspaces in $\mathcal{F}^{\prime}$. Double counting the pairs of subspaces $(A, B)$ where $B \in \mathcal{F}_{\frac{n+1}{2}}^{\prime}$ and $A$ is a $\frac{n-1}{2}$ dimensional subspace of $B$ we have $\left|N\left(\mathcal{F}_{\frac{n+1}{2}}^{\prime}\right)\right| \geqslant\left|\mathcal{F}_{\frac{n+1}{2}}^{\prime}\right|$.

Suppose $\left|N\left(\mathcal{F}_{\frac{n+1}{2}}^{\prime}\right)\right|>\left|\mathcal{F}_{\frac{n+1}{2}}^{\prime}\right|$, then we must have a pair of subspaces in the family related by containment (by our assumption that $\mathcal{F}^{\prime}$ has size $\left[\begin{array}{c}n \\ \frac{n+1}{2}\end{array}\right]_{q}$ ), but this contradicts Fact 26 and Lemma 24 .

Now suppose $\left|N\left(\mathcal{F}_{\frac{n+1}{2}}^{\prime}\right)\right|=\left|\mathcal{F}_{\frac{n+1}{2}}^{\prime}\right|$, then it follows from Lemma 27 that $\mathcal{F}_{\frac{n+1}{2}}^{\prime}$ is either empty or the complete level. This completes the proof of Theorem 8 .

Remark 28. Remark 25 also holds for Theorem 8.

Now we turn to the proof of Theorem 4. Clearly, the even case follows from Theorem 7. So we need to prove the case when $n$ is odd.

Proof of Theorem 4: By Remark 28, when $k=l=2$, the weaker condition for upper bound on $|\mathcal{F}|$ is $q^{2}-q-1>0$. This is true for $q \geqslant 2$, and this completes the proof of $\operatorname{La}_{q}^{*}(n,\{\mathrm{~V}, \Lambda\})=\left[\begin{array}{c}n \\ \frac{n+1}{2}\end{array}\right]_{q}$. Furthermore, the weaker condition for structure of $\mathcal{F}$ is $q^{2}-3 q+1>0$, and this inequality is true for $q \geqslant 3$.
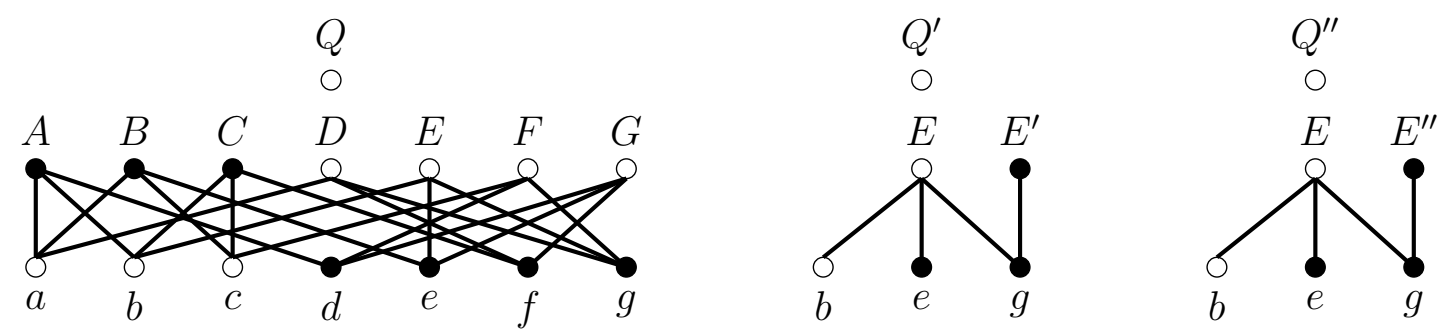

\section{$\stackrel{\circ}{P}$}

Figure 2: Small examples and illustration of the proof of Theorem 4.

For $q=2$, we can list all the cases for $n=3$, and there are two constructions which are not levels. (See Figure 2: $\{A, B, C, d, e, f, g\}$ (solid vertices) and $\{a, b, c, D, E, F, G\}$ (hollow vertices) are the two examples.)

Note that in this structure, there is a matching with 3 edges connecting 6 subspaces and a single isolated subspace. In Figure $2,\{A, d\},\{B, e\}$ and $\{C, f\}$ form the matching with 3 edges, and $g$ is the single isolated subspace. (Similarly, $\{D, a\},\{E, b\}$ and $\{F, c\}$ form the matching with 3 edges, and $G$ is the single isolated subspace.)

However, these constructions do not extend beyond the case $n>3$ for $q=2$. Similarly, we will prove the only induced $\{\mathrm{V}, \Lambda\}$-free family of maximum size is either $\left[\frac{V}{2}\right]_{q}$ or 
$\left[\begin{array}{c}V \\ \frac{n-1}{2}\end{array}\right]_{q}$ by three cases when $n>3$ and $q=2$. The first two cases $\operatorname{dim}(F) \geqslant \frac{n+1}{2}$ for all $F \in \mathcal{F}$ or $\operatorname{dim}(F) \leqslant \frac{n-1}{2}$ for all $F \in \mathcal{F}$ can be proved by Lemmas 17 and 18, since $q^{\left\lceil\frac{n}{2}\right\rceil} \geqslant 2$. Again for the third case, by Corollary 22, we may suppose that for every $F \in \mathcal{F}, \frac{n-1}{2} \leqslant \operatorname{dim}(F) \leqslant \frac{n+1}{2}$, and $\mathcal{F}_{\frac{n-1}{2}} \neq \emptyset$ and $\mathcal{F}_{\frac{n+1}{2}} \neq \emptyset$. Then, again by Lemma 27 and the assumption that $|\mathcal{F}|=\left[\begin{array}{c}n \\ \frac{n+1}{2}\end{array}\right]_{q}$, we can find two subspaces (say $d \subset A$ ) in $\mathcal{F}$, where $\operatorname{dim}(d)=\frac{n-1}{2}$ and $\operatorname{dim}(A)=\frac{n+1}{2}$.

Instead of using Lemma 24 to derive a contradiction, we must consider a more detailed argument. Suppose $\operatorname{dim}(Q)=\frac{n+3}{2}$ and $\operatorname{dim}(P)=\frac{n-3}{2}$ such that $P \subset d \subset A \subset Q$. We apply Fact 26 for the pair $(Q, P)$. Then we have 7 (that is, $q^{2}+q+1$ ) subspaces in $\mathcal{F}$ between $P$ and $Q$. Since $d$ and $A$ are not in the same level, without loss of generality, we can suppose that $A, B, C, d, e, f, g \in \mathcal{F}$ and $a, b, c, D, E, F, G \notin \mathcal{F}$ (as in Figure 2).

Since $n \geqslant 5$, we have $\left[\frac{n-1}{2}\right]_{q} \geqslant 3$ superspaces of dimension $\frac{n+3}{2}$ for every subspace of dimension $\frac{n+1}{2}$.

Thus, $E$ has two other $\frac{n+3}{2}$-dimensional superspaces: $Q^{\prime}$ and $Q^{\prime \prime}$. Now, we apply Fact 26 for the pairs $\left(Q^{\prime}, P\right)$ and $\left(Q^{\prime \prime}, P\right)$. Then we have 7 subspaces in $\mathcal{F}$ between $P$ and $Q^{\prime}\left(P\right.$ and $\left.Q^{\prime \prime}\right)$. (When we consider a $(\mathrm{V}, \Lambda)$-free family of size 7 between $P$ and $Q^{\prime}$ $\left(P\right.$ and $\left.Q^{\prime \prime}\right)$, the same argument applies as in the $n=3$ case above.) Clearly, there are only 3 subspaces $b, e$ and $g$ of $E$ containing $P$. (See Figure 2.) So these 3 subspaces $b, e$ and $g$ are also among the 7 total $\left(\frac{n-1}{2}\right)$-dimensional subspaces between $P$ and $Q^{\prime}(P$ and $\left.Q^{\prime \prime}\right)$. Since there are 7 total $\left(\frac{n-1}{2}\right)$-dimensional subspaces and 7 total $\left(\frac{n+1}{2}\right)$-dimensional subspaces between $P$ and $Q^{\prime}\left(P\right.$ and $\left.Q^{\prime \prime}\right)$, by the assumption $b \notin \mathcal{F}$ and $e, g \in \mathcal{F}$, all 7 subspaces in $\mathcal{F}$ between $P$ and $Q^{\prime}\left(P\right.$ and $\left.Q^{\prime \prime}\right)$ cannot form a level, and so they form a matching of 3 edges and a single isolated subspace.

Now, we will show that $e$ is the single isolated subspace. Suppose not, say $B^{\prime}$ is the superspace of $e$ in $\mathcal{F}$ between $P$ and $Q^{\prime}\left(P\right.$ and $\left.Q^{\prime \prime}\right)$. We have $B \neq B^{\prime}$, since otherwise $B, E, Q$ and $Q^{\prime}\left(Q^{\prime \prime}\right)$ form a butterfly. However, by the assumption $B \in \mathcal{F}$, we have that $B^{\prime}, e$ and $B$ will form an induced $\mathrm{V}$, since $\operatorname{dim}(B)=\operatorname{dim}\left(B^{\prime}\right)$ and $B \neq B^{\prime}$. Note that $e$ is the single isolated subspace implies that $g$ is in an edge of the matching formed by subspaces in $\mathcal{F}$ between $P$ and $Q^{\prime}\left(P\right.$ and $\left.Q^{\prime \prime}\right)$. Thus, there exist $E^{\prime} \subset Q^{\prime}$ and $E^{\prime \prime} \subset Q^{\prime \prime}$ in $\mathcal{F}$ such that $g \subset E^{\prime}, E^{\prime \prime}$. Note that $E^{\prime} \neq E^{\prime \prime}$, otherwise $E, E^{\prime}, Q^{\prime}$ and $Q^{\prime \prime}$ would form a butterfly. It follows that $g, E^{\prime}$ and $E^{\prime \prime}$ form an induced $\mathrm{V}$ in $\mathcal{F}$, a contradiction.

\section{General LYM-type lemma}

Let $V$ be an $n$-dimensional vector space over a finite field $\mathbb{F}_{q}$, where $q$ is a prime power. Let $\mathcal{H}$ be a family of subspaces of $V$. We say that $\mathcal{H}$ is simple if there is a basis $\left\{v_{1}, v_{2}, \ldots, v_{n}\right\}$ of $V$ such that $\mathcal{H} \subseteq\left\{\operatorname{span}(S): S \in 2^{\left\{v_{1}, v_{2}, \ldots, v_{n}\right\}}\right\}$, where $\operatorname{span}(S)$ is the subspace spanned by the basis vectors in $S$. For any poset $P$, let $\alpha(\mathcal{H}, P)$ denote the maximum size of a $P$-free subfamily of $\mathcal{H}$. We denote by $N_{i}(\mathcal{H})$ the number of $i$-dimensional subspaces in $\mathcal{H}$.

We now present a general LYM-type lemma. The proof comes from adopting the methods from [8] to a vector space setting. 
Theorem 29. Let $\mathcal{F}$ be a $P$-free family of subspaces of $V$, and let $\mathcal{H}$ be a simple family of subspaces of $V$, then

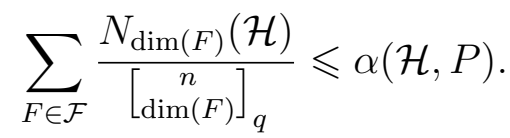

In particular, if $N_{k}(\mathcal{H})=N$ for a given integer $N$ and all $k$, then

$$
\sum_{F \in \mathcal{F}} \frac{1}{\left[\begin{array}{c}
n \\
\operatorname{dim}(F)]_{q}
\end{array}\right.} \leqslant \frac{\alpha(\mathcal{H}, P)}{N} .
$$

Proof: Consider a set $H \in \mathcal{H}$, and without loss of generality assume

$$
H=\operatorname{span}\left(\left\{v_{1}, v_{2}, \ldots, v_{r}\right\}\right) .
$$

Let $F$ be a subspace of $V$ of dimension $r$. Consider maps $\pi$ which replace the basis $\left\{v_{1}, v_{2}, \ldots, v_{n}\right\}$ of $V$ with an arbitrary basis $\left\{w_{1}, w_{2}, \ldots, w_{n}\right\}$ of $V$ and assign $\pi\left(v_{i}\right)=w_{i}$ for $i \in[n]$. For a set $H=\operatorname{span}\left(\left\{v_{i_{1}}, v_{i_{2}}, \ldots, v_{i_{r}}\right\}\right) \in \mathcal{H}$, let

$$
H^{\pi}=\operatorname{span}\left(\left\{\pi\left(v_{i_{1}}\right), \pi\left(v_{i_{2}}\right), \ldots, \pi\left(v_{i_{r}}\right)\right\}\right),
$$

and set $\mathcal{H}^{\pi}=\left\{H^{\pi}: H \in \mathcal{H}\right\}$.

We will double count pairs $(F, \pi)$ such that $F \in \mathcal{F}$ and $F \in \mathcal{H}^{\pi}$. Suppose $F \in \mathcal{F}$ and $H \in \mathcal{H}^{\pi}$ both have dimension $r$. The number of $\pi$ such that $F \in \mathcal{H}^{\pi}$ is

$$
\left(q^{r}-1\right)\left(q^{r}-q\right) \ldots\left(q^{r}-q^{r-1}\right)\left(q^{n-r}-1\right)\left(q^{n-r}-q\right) \ldots\left(q^{n-r}-q^{n-r-1}\right) .
$$

Observe that if for two distinct $H_{1}, H_{2} \in \mathcal{H}$ we have $F=H_{1}^{\pi_{1}}$ and $F=H_{2}^{\pi_{2}}$, then $\pi_{1} \neq \pi_{2}$. It follows that for each $F \in \mathcal{F}$, there are

$$
\left(q^{r}-1\right)\left(q^{r}-q\right) \ldots\left(q^{r}-q^{r-1}\right)\left(q^{n-r}-1\right)\left(q^{n-r}-q\right) \ldots\left(q^{n-r}-q^{n-r-1}\right) N_{\operatorname{dim}(F)}(\mathcal{H})
$$

mappings $\pi$ such that $F \in \mathcal{H}^{\pi}$. Thus, on the one hand, the number of pairs $(F, \pi)$ is

$$
\sum_{F \in \mathcal{F}}\left(q^{r}-1\right)\left(q^{r}-q\right) \ldots\left(q^{r}-q^{r-1}\right)\left(q^{n-r}-1\right)\left(q^{n-r}-q\right) \ldots\left(q^{n-r}-q^{n-r-1}\right) N_{\operatorname{dim}(F)}(\mathcal{H}),
$$

or equivalently,

$$
\sum_{F \in \mathcal{F}}[\operatorname{dim}(F)]_{q} ![n-\operatorname{dim}(F)]_{q} !(q-1)^{n} N_{\operatorname{dim}(F)}(\mathcal{H}) .
$$

Now suppose we fix a mapping $\pi$. Since $\mathcal{H}$ and $\mathcal{H}^{\pi}$ are isomorphic as posets with respect to the subspace relation, we have at most $\alpha(\mathcal{H}, P)$ many $F \in \mathcal{F}$ such that $F \in \mathcal{H}^{\pi}$. Since the total number of mappings $\pi$ is

$$
\left(q^{n}-1\right)\left(q^{n}-q\right) \ldots\left(q^{n}-q^{n-1}\right),
$$


we have an upper bound on the number of pairs $(F, \pi)$ of

$$
\left(q^{n}-1\right)\left(q^{n}-q\right) \ldots\left(q^{n}-q^{n-1}\right) \alpha(\mathcal{H}, P)
$$

or equivalently,

$$
[n]_{q} !(q-1)^{n} \alpha(\mathcal{H}, P) .
$$

Combining (2) and (3), we have

$$
\sum_{F \in \mathcal{F}}[\operatorname{dim}(F)]_{q} ![n-\operatorname{dim}(F)]_{q} ! N_{\operatorname{dim}(F)}(\mathcal{H}) \leqslant[n]_{q} ! \alpha(\mathcal{H}, P),
$$

and rearranging yields the desired inequality.

Remark 30. The exact same arguments can be carried out to prove the analogous result when we forbid $P$ as an induced subposet.

Now, we use Lemma 29 to prove vector space versions of Theorems 10, 11, 13 and 14. We remark that the vector space of Theorem 10 was conjectured by Shahriari and Yu.

Conjecture 31 (Shahriari and $\mathrm{Yu}[13]$ ). Let $k \geqslant 1$ and $n \geqslant k+1$, then

$$
\operatorname{La}_{q}\left(n,\left\{Y_{k}, Y_{k}^{\prime}\right\}\right)=\Sigma_{q}(n, k)
$$

We will show that even the induced version of this conjecture holds.

Theorem 32. Let $k \geqslant 1$ and $n \geqslant k+1$, then

$$
\operatorname{La}_{q}^{*}\left(n,\left\{Y_{k}, Y_{k}^{\prime}\right\}\right)=\Sigma_{q}(n, k)
$$

Let $\left\{v_{1}, v_{2}, \ldots, v_{n}\right\}$ be a basis of $V$ and $\mathcal{I}_{n}$ be the family of subspaces formed by arranging the basis $\left\{v_{1}, v_{2}, \ldots, v_{n}\right\}$ in order around a circle and taking those subspaces (excluding $\{0\}$ and $V$ ) which are spanned by vectors along this cyclic arrangement.

Lemma 33 (Tompkins and Wang [16]). Let $\alpha^{*}\left(\mathcal{I}_{n}, Y_{k}, Y_{k}^{\prime}\right)$ be the maximum size of an induced $\left\{Y_{k}, Y_{k}^{\prime}\right\}$-free subfamily of $\mathcal{I}_{n}$, then

$$
\alpha^{*}\left(\mathcal{I}_{n}, Y_{k}, Y_{k}^{\prime}\right)=k n
$$

Proof of Theorem 32: Clearly, $N_{i}\left(\mathcal{I}_{n}\right)=n$ for all $i$. If $\mathcal{F}$ is an induced $\left\{Y_{k}, Y_{k}^{\prime}\right\}$-free family such that $\{0\}, V \notin \mathcal{F}$, it follows from Remark 30 that

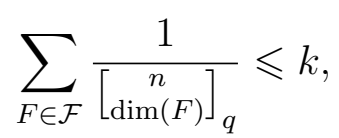

and so

$$
|\mathcal{F}| \leqslant \Sigma_{q}(n, k)
$$


Otherwise, if $\{0\}, V \in \mathcal{F}$, we can assume the result is true for $k-1$. Note that the base case $k=1$ is proved by Theorem 4 . Then it follows that

$$
|\mathcal{F}| \leqslant \Sigma_{q}(n, k-1)+2 \leqslant \Sigma_{q}(n, k),
$$

since $\mathcal{F}$ is induced $\left\{Y_{k-1}, Y_{k-1}^{\prime}\right\}$-free.

Now we may assume that $\{0\} \in \mathcal{F}$ and $V \notin \mathcal{F}$. Let $\mathcal{G}=\mathcal{F} \backslash\{\{0\}\}$. If $|\mathcal{G}| \leqslant \Sigma_{q}(n, k)-1$, then $|\mathcal{F}| \leqslant \Sigma_{q}(n, k)$. So we may assume $|\mathcal{G}|=\Sigma_{q}(n, k)$, and $\mathcal{G}$ is a subfamily of the $k$ (or $k+1$ ) largest levels in the linear lattice. If $n \neq k$ modulo 2 , then $\mathcal{G}$ is uniquely determined (i.e., the largest $k$ levels), and it is easy to find an induced $Y_{k}$ in $\mathcal{F}$. Indeed, we can find an induced $Y_{k-1}$ in $\mathcal{G}$, which together with $\{0\}$ form an induced $Y_{k}$. If $n=k$ modulo 2 , then $\mathcal{G}$ is a subfamily of the $k+1$ largest levels $\mathcal{L}_{1}, \mathcal{L}_{2}, \ldots, \mathcal{L}_{k+1}$. If $\mathcal{G} \cap \mathcal{L}_{1}=\emptyset$, then we can find an induced $Y_{k}$ as in the previous case. Otherwise, let $L_{1} \in \mathcal{G} \cap \mathcal{L}_{1}$, then find an induced $Y_{k-2}$ in $\mathcal{L}_{2} \cup \cdots \mathcal{L}_{k+1}$ such that $L_{1}$ is a subspace of every subspace in this $Y_{k-2}$. It is easy to see that $\{0\}, L_{1}$ together with this $Y_{k-2}$ form an induced $Y_{k}$ in $\mathcal{F}$.

We now recall some other structures which have been used in double counting arguments for forbidden poset problems.

Definition 34. Let $\emptyset=A_{0} \subset A_{1} \subset \cdots \subset A_{n-1} \subset A_{n}=[n]$ be a maximal chain in the $n$-element Boolean lattice. Then the $k$-interval chain defined from this maximal chain is given by $\left[A_{0}, A_{k}\right] \cup\left[A_{1}, A_{k+1}\right] \cup \cdots \cup\left[A_{n-k}, A_{n}\right]$. A 2-interval chain is called a double chain.

Lemma 35 (Burcsi and Nagy [1]). Given a double chain $\mathcal{D}$, we have $N_{i}(\mathcal{D})=2$ for every $i$ and

$$
\alpha(\mathcal{D}, P)=|P|+h(P)-2 .
$$

Lemma 36 (Grósz, Methuku and Tompkins [8]). Given a k-interval chain $\mathcal{C}_{k}$, we have $N_{i}\left(\mathcal{C}_{k}\right)=k$ for every $i$ and

$$
\alpha\left(\mathcal{C}_{k}, P\right)=\frac{k}{2^{k-1}}\left(|P|+(3 k-5) 2^{k-2}(h(P)-1)-1\right) .
$$

By Lemma 29, the vector space versions of Theorems 13 and 14 follows from the above two lemmas, respectively.

Remark 37. In proving the vector space version of Theorem 14 from the Lemma 36, we proceed exactly as in the corresponding proof in [8] replacing each binomial coefficient with the corresponding $q$-binomial, and verify that all the estimates still hold.)

Theorem 38. For any poset $P$, when $n$ is sufficiently large, we have

$$
\operatorname{La}_{q}(n, P) \leqslant\left(\frac{|P|+h(P)}{2}-1\right)\left[\begin{array}{c}
n \\
\left\lfloor\frac{n}{2}\right\rfloor
\end{array}\right]_{q} .
$$

Theorem 39. For any poset $P$, when $n$ is sufficiently large, we have

$$
\mathrm{La}_{q}(n, P) \leqslant \frac{1}{2^{k-1}}\left(|P|+(3 k-5) 2^{k-2}(h(P)-1)-1\right)\left[\begin{array}{c}
n \\
\left\lfloor\frac{n}{2}\right\rfloor
\end{array}\right]_{q},
$$

for any fixed $k$. 


\section{Acknowledgements}

The authors would like to thank Gyula O.H. Katona for some useful discussions and also the anonymous referee whose remarks, in particular, simplified the proof of Theorem 8 (an earlier version of the manuscript invoked a stronger result of Chowdury and Patkós [3]).

\section{References}

[1] P. Burcsi and D. Nagy, The method of double chains for largest families with excluded subposets, Electron. J. Graph Theory Appl. (EJGTA) 1(1) (2013), 40-49.

[2] H. Chen and W. Li, A Note on the Largest Size of Families of Sets with a Forbidden Poset, Order, 31 (2014), 137-142.

[3] A. Chowdhury and B. Patkós, Shadows and intersections in vector spaces, J. Combin. Theory Ser. A, 117 (2010), 1095-1106.

[4] A. De Bonis and Gy. Katona, Largest families without an r-fork, Order 24 (2007) 181-191.

[5] A. De Bonis, Gy. Katona and K. Swanepoel, Largest family without $A \cup B \subset C \cap D$, J. Combin. Theory Ser. A, 111 (2005), 331-336.

[6] D. Gerbner, The covering lemma and $q$-analogues of extremal set theory problems, arXiv:1905.06994, (2019).

[7] D. Gerbner, A. Methuku, D.T. Nagy, B. Patkós and M. Vizer, Forbidding rank-preserving copies of a poset, Order (2019), 1-10, doi:10.1007/s11083-019-09484-5.

[8] D. Grósz, A. Methuku, and C. Tompkins, An improvement of the general bound on the largest family of subsets avoiding a subposet, Order 34 (2017), 113-125.

[9] S. Ghassan and S. Shahriari, Diamond-free subsets in the linear lattices, Order 31.3 (2014), 421-433.

[10] Gy. Katona and T. Tarján, Extremal problems with excluded subgraphs in the $n$ cube, Graph theory (Lagów, 1981), Lecture Notes in Math., vol. 1018, Springer, Berlin, (1983), 84-93.

[11] R. Martin, A. Methuku, A. Uzzell, and S. Walker, A simple discharging method for forbidden subposet problems, arXiv:1710.05057, (2017).

[12] A. Methuku and C. Tompkins, Exact forbidden subposet results using chain decompositions of the cycle, The Electronic Journal of Combinatorics, 22(4), \#P4.29 (2015).

[13] S. Shahriari and S. Yu, Avoiding Brooms, Forks, and Butterflies in the Linear Lattices, arXiv:1807.06259, (2018).

[14] E. Sperner, Ein Satz über Untermengen einer endlichen Menge, Mathematische Zeitschrift, (1928), 544-548. 
[15] H. Thanh, An extremal problem with excluded subposets in the Boolean lattice, Order 15 (1998) 51-57.

[16] C. Tompkins and Y. Wang, On an extremal problem involving a pair of forbidden posets, arXiv:1710.10760, (2017). 